\title{
Regulation of tissue iron homeostasis: the macrophage "ferrostat"
}

\author{
Nathan C. Winn, ${ }^{1}$ Katrina M. Volk, ${ }^{1}$ and Alyssa H. Hasty, \\ 'Department of Molecular Physiology and Biophysics, Vanderbilt University School of Medicine, Nashville, Tennessee, USA \\ ${ }^{2}$ VA Tennessee Valley Healthcare System, Nashville, Tennessee, USA.
}

Iron is an essential element for multiple fundamental biological processes required for life; yet iron overload can be cytotoxic. Consequently, iron concentrations at the cellular and tissue level must be exquisitely governed by mechanisms that complement and fine-tune systemic control. It is well appreciated that macrophages are vital for systemic iron homeostasis, supplying or sequestering iron as needed for erythropoiesis or bacteriostasis, respectively. Indeed, recycling of iron through erythrophagocytosis by splenic macrophages is a major contributor to systemic iron homeostasis. However, accumulating evidence suggests that tissue-resident macrophages regulate local iron availability and modulate the tissue microenvironment, contributing to cellular and tissue function. Here, we summarize the significance of tissue-specific regulation of iron availability and highlight how resident macrophages are critical for this process. This tissue-dependent regulation has broad implications for understanding both resident macrophage function and tissue iron homeostasis in health and disease.

Conflict of interest: The authors have declared that no conflict of interest exists.

Copyright: $\odot$ 2020, American Society for Clinical Investigation.

Reference information: /CI Insight. 2020;5(2):e132964.

https://doi.org/10.1172/jici.

insight.132964.

\section{Introduction}

Iron is an essential element for various fundamental biological processes necessary for life. In mammals, iron is incorporated into proteins that are central for cellular respiration, DNA synthesis, proliferation, xenobiotic metabolism, host defense, and cell signaling. The importance of iron in physiology is evidenced by the pathophysiological consequences triggered by iron deficiency, including impairments in cognitive development, birth defects, cardiovascular diseases, and a host of other health complications $(1,2)$. Iron overload is also detrimental to health, leading to adverse manifestations in multiple tissues, including the heart, liver, adipose, brain, muscle, and pancreas, and is implicated in the pathogenesis of several metabolic (e.g., type 2 diabetes, nonalcoholic steatohepatitis [NASH]) and neurodegenerative diseases (e.g., Alzheimer's disease and Parkinson's disease) $(3,4)$. Excess intracellular labile iron interacts with ROS that are produced in aerobic conditions, leading to cell and tissue damage via Fenton chemistry (5). Thus, iron concentrations at the cellular and tissue level must be exquisitely governed by mechanisms that complement and fine-tune systemic control — including uptake, transport, storage, and export — in order to maintain local and systemic iron homeostasis.

Macrophages (Mфs), the principal cells responsible for handling iron in mammals, are present in all tissues and are pertinent to tissue homeostatic function (6-10). $M \phi s$ are highly plastic in response to the tissue niche, acquiring rapid polarization on a spectrum from an M1-like proinflammatory to M2-like tissue repair phenotype (reviewed in refs. 11, 12). Although classically appreciated for their surveillance role, tissue-resident, self-renewing $\mathrm{M} \phi \mathrm{s}$ have crucial homeostatic functions - including tissue remodeling, pathogen recognition, cell repair, and phagocytic clearance of apoptotic and senescent cells - in virtually all tissues (13-15). In addition to performing basic housekeeping functions, tissue $\mathrm{M} \phi \mathrm{s}$ also help maintain and/or restore homeostatic balance in response to various perturbations. For example, during ischemia or tissue hypoxia, M $\phi$ s act as angiogenic accessory cells that support endothelial cell proliferation and vessel sprouting to enhance perfusion (16). Notably, the accessory role of $\mathrm{M} \phi \mathrm{s}$ in supporting metabolically active parenchymal cells is linked with iron handling. Indeed, a postulate gaining traction is that tissue $M \phi s$ are so-called ferrostats that sense and respond to local tissue iron needs, thereby regulating the tissue microenvironment. One example of this tenet is a specialized population of adipose tissue (AT) M $\phi s$ (ATM $\phi s$ ) - previously identified by our group - that are iron-rich $\left(\mathrm{MFe}^{\mathrm{hi}}\right)$ and have the intrinsic capacity to take up excess iron, and thereby protect adipocytes from iron overload $(17,18)$. These iron-cycling $M \phi s$ are not lim- 
ited to AT, and mounting evidence reveals their presence and homeostatic functions in multiple tissues and organs. Accordingly, this Review highlights the importance of tissue-specific regulation of iron availability and summarizes how resident $\mathrm{M} \phi$ s are fundamental to this homeostatic circuit.

\section{Overview of $М \phi$ iron flux}

Systemically, iron is redistributed for two primary reasons: (i) it is supplied for erythropoiesis and (ii) it is sequestered for bacteriostasis $(9,19,20)$. However, tissue-resident $\mathrm{M} \phi$ s may also serve as a bioavailable iron storage compartment that is poised for mobilization based on local tissue needs (see "Tissue M $\phi s$ regulate iron homeostasis and tissue function"). On a cellular level, iron is required for basic processes that contribute to cell growth, maintenance, repair, and even cell death. Thus, local iron availability must be tightly controlled to ensure cellular homeostasis. Several recent and excellent reviews have been published describing in-depth mechanisms for intestinal iron absorption, systemic transport, and cellular import/export (6, 21-23). In this section, we provide a brief overview of multiple key players that contribute to tissue iron cycling by regulating cellular iron import, catabolism/storage, and export (Figure 1).

$\mathrm{M} \phi$ s take up iron or iron-containing molecules via receptors such as transferrin $(\mathrm{Tf})$ receptor protein 1 (TfR1; also known as CD71), LDL-related receptor 1 (LRP1; also known as CD91), and the hemoglobin-haptoglobin receptor (CD163), which bind transferrin-bound iron, heme-hemopexin (Hx-heme), and hemoglobin-haptoglobin ( $\mathrm{Hb}-\mathrm{Hp})$, respectively; as well as via phagocytosis of erythrocytes and other cells. Plasma ferric iron $\left(\mathrm{Fe}^{3+}\right)$ is scavenged by apo-Tf, and the resultant holo-Tf is endocytosed after binding TfR1 via clathrin-mediated endocytosis. Within the endosomal compartment, $\mathrm{Fe}^{3+}$ is reduced to ferrous iron $\left(\mathrm{Fe}^{2+}\right)$ by the six-transmembrane epithelial antigen of prostate (STEAP3) enzyme before entering the cytosolic labile iron pool (LIP) via the assistance of divalent metal transporter 1 (DMT1) (24). Extracellular non-Tf-bound $\mathrm{Fe}^{2+}$ iron (NTBI) is imported via plasma membrane-localized DMT1 and/or the zinc transporter ZRT/IRT-like protein 14 (ZIP14) and can be directly incorporated into the cytosolic LIP. The intracellular LIP is destined for storage, export, or trafficking (Figure 1). Although some of the LIP is used by $\mathrm{Fe}^{2+}$-dependent proteins in the cytosol, a large majority of iron is trafficked to the mitochondria and incorporated into heme and Fe-S clusters that assist with electron transport and enzyme catalysis (25). Heme oxygenase 1 (HO-1) breaks down heme-iron into $\mathrm{Fe}^{2+}$ and two antiinflammatory products, biliverdin and carbon monoxide. $\mathrm{Fe}^{2+}$ iron from the LIP that is not metabolized or exported is then stored within the cytosol in a nontoxic heteropolymer comprising ferritin heavy (FtH1) and ferritin light (FtL) chains that cage up to 4500 atoms of iron (26). FtH1 has inherent ferroxidase activity, which is required for iron mineralization into the ferritin nanocage (27). Thus, global FtH1 ablation is embryonically lethal (28), whereas loss-of-function mutations in the FtL-encoding gene or frameshift mutations that alter the FtL C-terminus have been described in humans $(29,30)$. When the LIP is low, intracellular iron can be mobilized via ferritin degradation by nuclear receptor coactivator 4 (NCOA4) in a process termed "ferritinophagy" (i.e., lysosomal degradation of ferritin) (31). NCOA4 silencing in mice results in hypo-ferritinophagy and anemia due to an inability to mobilize iron from intracellular ferritin stores (32). Thus, the role of ferritinophagy is critical for intracellular iron mobilization, and perturbation of this process is linked with several pathologies reviewed elsewhere (31, 33-35).

Cellular iron is exported as $\mathrm{Fe}^{2+}$ via the transmembrane protein ferroportin (Fpn), which contains two lobes that form a central cavity and transition between inward and outward conformations, accounting for export activity (23). Following Fpn-mediated export, the ferroxidase enzyme ceruloplasmin (Cp), localized in the extracellular space, oxidizes $\mathrm{Fe}^{2+}$ to $\mathrm{Fe}^{3+}$, which is required for $\mathrm{Tf}$ binding and Tf-mediated transport throughout the body (36). Fpn is highly expressed in duodenal enterocytes, hepatocytes, and macrophages (23). It is fundamental for iron export, such that global Fpn deletion abolishes iron export and is embryonically lethal $(37,38)$. Deletion of $F p n$ in all tissues, except extraembryonic visceral endoderm and placenta, produces viable pups; however, these animals display growth retardation and anemia shortly after birth (38). Fpn transcription is activated by nuclear factor erythroid 2-like factor 2 (NRF2) and inhibited by Btb and Cnc homology 1 (BACH1) (23). In addition, specific pathogen-associated molecular patterns (PAMPs) bind to TLRs and subsequently suppress Fpn transcription - a mechanism important for host defense (39). Hepcidin, a protein produced and secreted principally by the liver, inhibits iron efflux by embedding itself within the center of Fpn and promoting Fpn receptor internalization, ubiquitination, and degradation (6). For example, a single exogenous bolus of hepcidin markedly lowers serum iron concentrations, and it is thought that $\mathrm{M} \phi \mathrm{s}$ are more sensitive to the suppressive effects of hepcidin on Fpn-mediated iron export compared with other 


\section{\begin{tabular}{|l|l|l|l|l|l} 
Uptake & Catabolism & Storage & Export \\
\hline
\end{tabular}}

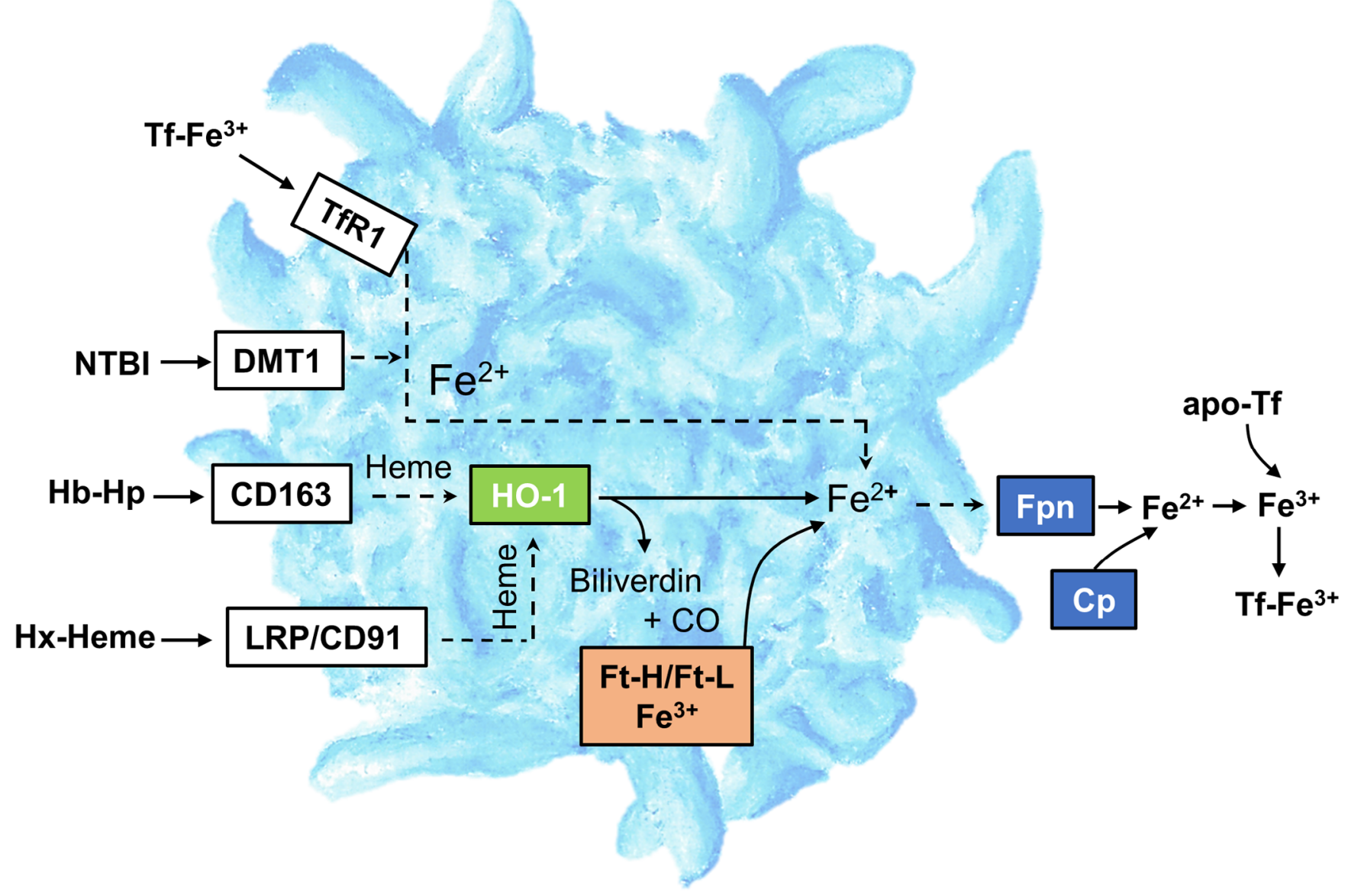

Figure 1. Overview of $\mathbf{M} \phi$ iron metabolism. The uptake of Tf-bound $\mathrm{Fe}^{3+}$ is mediated by TfR1. CD163 and LRP1 (also known as CD91) mediate the uptake of $\mathrm{Hb}-\mathrm{Hp}$ and $\mathrm{Hx}$-heme, respectively. Intracellular heme-Fe (extracted from $\mathrm{Hb}$ - $\mathrm{Hp}$ and $\mathrm{Hx}$-heme) is catabolized by $\mathrm{HO}-1$ for incorporation into the cellular ferritin pool or trafficked into the mitochondria. Non-Tf-bound iron (NTBI) is imported via DMT1. Iron export by Fpn is facilitated by Cp, which oxidizes Fe ${ }^{2+}$ to $\mathrm{Fe}^{3+}$ to allow sequestration by apo-Tf. Tf, transferrin; TfR1, transferrin receptor 1; LRP/CD91, LDL-related receptor 1/CD91; DMT1, divalent metal transporter 1; Hb-Hp, hemoglobin-haptoglobin; Hx-heme, hemopexin-heme; HO-1, heme oxygenase 1; Ft- $\mathrm{H}$, ferritin heavy chain 1; Ft-L, ferritin light chain; Fpn, ferroportin; Cp, ceruloplasmin.

cell types $(40,41)$. Indeed, Fpn protein expression is suppressed to a greater extent after 24 hours of hepcidin treatment in THP-1 M $\phi$ s compared with intestinal epithelial Caco-2 cells. Furthermore, hepcidin treatment lowers Fpn immunoreactivity to a greater extent in $\mathrm{M} \phi$-rich splenic red pulp than in white pulp spleen sections (41). Thus, hepcidin plays an important role as a modulator of global iron cycling by exerting a high degree of regulatory control over duodenal iron absorption and Fpn-mediated macrophage iron export.

While according to current dogma, iron leaves the cell principally via Fpn transport, it is worth noting that some data suggest that cellular ferritin efflux may be a mechanism for $\mathrm{Fe}^{3+}$ export (42-45). Moreover, a recent study proposed an alternative iron export pathway, via GAPDH-mediated retro-endocytosis of iron-loaded $\mathrm{Tf}$ (46), and an important but underappreciated iron-release mechanism employed by tumor-associated M $\phi s$ (TAMs) to kill cancer cells has been described. In this context, M1-like TAMs produce NO via iNOS that evokes iron efflux from cancer cells (47-49). Mechanistically, NO, in addition to glutathione, binds labile iron in cancer cells, leading to formation of dinitrosyl diglutathionyl iron complexes (DNDGICs; also termed DNICs). DNICs are long-lived forms of NO-Fe that can undergo subsequent release from affected cancer cells by multidrug resistance-associated protein 1 (MRP1), leading to cancer cell iron depletion and diminished proliferation. Conversely, DNICs can be sequestered by glutathione- $S$-transferase P1-1 expressed in cancer cells and/or M $\phi s(47,48)$. Taken together, there appear to 
be several redundant iron efflux mechanisms that may be disease specific; yet experimentally, cell-specific modulation of Fpn function is a useful tool to study targeted iron overload or perturbed iron trafficking.

Because it is essential for life and at the same time has poor bioavailability, iron is recycled - largely through the erythrocyte hemoglobin cycle (50). Indeed, de novo synthesis of hemoglobin consumes up to $25 \mathrm{mg}$ iron per day, with intestinal iron absorption only accounting for 1-2 mg daily (6). Hence, iron recycling via phagocytosis of senescent red blood cells by splenic red pulp M $\phi s$ (RPM $\phi s)$ and liver Kupffer cells (KCs) largely maintains systemic iron concentrations (51). This systemic iron recycling prevents damage from excess iron, heme, and hemoglobin deposition in organs caused by injured or dying red blood cells. Of note, heme-responsive gene 1 (HRG1) appears essential for heme transport from the phagolysosome to the cytoplasm during erythrophagocytosis and is considered necessary for heme recycling by $\mathrm{M} \phi \mathrm{s}(52,53)$. RPM $\phi$ s endocytose senescent erythrocytes and recycle the iron through $\mathrm{HO}-1$ breakdown and Fpn export, delivering it in a Tf-bound form to bone marrow for erythropoiesis (54). In fact, the specialized function of $\mathrm{RPM} \phi s$ is unique among splenic $\mathrm{M} \phi s$, such that an additional transcription factor, SpiC, is required for the development of these cells (55). Since dietary iron absorption is insufficient to sustain erythropoiesis, $\mathrm{RPM} \phi$ recycling of heme iron by HO-1 and its release by Fpn is critical $(37,56,57)$.

\section{М $\Phi$ polarization and iron handling}

$\mathrm{M} \phi$ polarization has been historically simplified to a binary categorization - existing in either an M1 proinflammatory or M2 antiinflammatory state. Although this is useful for generic classification, M $\phi$ s exhibit a spectrum of activation states in vivo (11). Nonetheless, it is recognized that even if classified according to the simplified M1/M2 nomenclature, M $\phi$ polarization modulates iron handling (58-60). Indeed, the high degree of iron cycling from M2 M $\$$ s may be related to their role in tissue repair and angiogenesis. These IL-4-activated $\mathrm{M} \phi \mathrm{s}$ are characterized by high expression of CD163, low expression of ferritin, and high expression of Fpn. In contrast, M1 polarization generally favors an iron storage phenotype, with increased expression of ferritin and decreased expression of Fpn, coinciding with bactericidal activity and $\mathrm{M} \phi$ cytokine production and immunostimulation $(58,61)$. Thus, there appears to be a close link between iron and $\mathrm{M} \phi$ polarization, such that the presence of iron and polarization modulate one another.

\section{Tissue $M \phi s$ regulate iron homeostasis and tissue function}

An intrinsic property of tissue-resident $\mathrm{M} \phi \mathrm{s}$ is their ability to maintain self-proliferation throughout adulthood, independent of monocyte recruitment $(62,63)$. Accumulating data suggest that tissue $M \phi s$ are ferrostats that sense and then regulate iron availability in the local microenvironment, contributing to cellular/tissue function. Importantly, this fine-tuning would allow for local control of iron homeostasis in response to the tissue's needs, which may be independent of systemic iron handling. Indeed, iron is now thought to be spatially regulated in various tissues on a microenvironmental scale and in a time-dependent manner - such as during wound repair (60-63), wherein resident M2-like $\mathrm{M} \phi s$ are largely responsible for the fine-tuned iron uptake and release necessary for proper restoration. Thus, the ferrostat notion has broad implications for understanding resident $\mathrm{M} \phi$ function and tissue iron homeostasis in both health and disease (64-67). In this section, we summarize tissue-specific regulation of iron by resident $\mathrm{M} \phi \mathrm{s}$ in physiological and/or pathological states.

Liver. The liver is a central hub in systemic iron metabolism, as it is the principal producer of hepcidin and transferrin (8). Many of the iron-handling functions of the liver are modulated and directed by resident KCs. Estimates suggest that these nonmigratory KCs constitute the largest tissue-resident $\mathrm{M} \phi$ population in the body $(68)$. KCs are yolk sac derived $(69,70)$ or arise from fetal hematopoietic stem cells, and reside within liver sinusoids (71). Given their hepatic residence, KCs are exposed to a rich supply of nutrients, invading pathogens and bacteria, and exogenous drugs and toxins. Various substances, including LPS, complement factors, fungi, and bacteria, activate KCs (72).

Importantly, KCs highly express iron metabolism-associated genes and are transcriptionally regulated by SpiC and NRF2 (73). The location within sinusoids positions KCs as the primary cells that recycle iron released from nearby senescent erythrocytes, which may assist in dampening hepatocyte iron overload (74). Aberrant KC activation leads to increased proinflammatory cytokine release and subsequent hepatocyte hepcidin production, resulting in an overall decrease in iron export from the liver. This decreased iron export into systemic circulation contributes to a state of acute anemia (75). Another study supports that KCs exert an inhibitory effect on hepatocyte hepcidin expression in the absence of inflammation (76), implying that KCs can bidirectionally regulate hepatic iron content in an inflammation-dependent manner. 
Spleen. RPM $\phi$ s in the spleen play a vital role in recycling iron from senescent erythrocytes. As noted, $\mathrm{RPM} \phi \mathrm{s}$ are regulated by the transcription factor SpiC (55) and depend on HO-1 activity for intracellular heme breakdown and free iron release (77). Heme itself may induce SpiC expression via degradation of the transcriptional repressor BACH1 (78). SpiC-knockout mice lack RPM $\phi$ s and accumulate ferritin in the splenic red pulp, leading to splenomegaly (55), indicating that the RPM $\phi$ s are necessary for iron recycling. Interestingly, a subpopulation of $\mathrm{F} 4 / 80^{\mathrm{hi}} \mathrm{Mac}-1^{\text {lo }} \mathrm{RPM} \phi \mathrm{s}$ has been implicated in regulating an excessive immune response by dampening $\mathrm{CD}^{+} \mathrm{T}$ cell activity (79), supporting the concept that these $\mathrm{M} \phi \mathrm{s}$ are tissue-regulatory cells. However, the immunomodulatory role of RPM $\phi$ s requires further investigation.

Bone and bone marrow. Multipotent hematopoietic stem cells within bone marrow exert master regulation over hematopoiesis and consequently govern erythropoiesis, with iron being a fundamental element in these processes. A specialized population of resident erythroid island $M \phi s$ supports erythropoiesis ( 80 , 81), and their differentiation is dependent on SpiC (78). A running hypothesis is that erythroid island $\mathrm{M} \phi \mathrm{s}$ serve as iron-rich nurse cells that support erythropoiesis. Indeed, recent data indicate that erythroid island Mфs highly express iron-cycling machinery including TfR1, HO-1, and Fpn (82). While transcriptomic analyses have provided insights into the potential mechanistic pathways by which erythroid island $\mathrm{M} \phi \mathrm{s}$ support erythropoiesis, additional studies are needed to determine the explicit function of this unique $\mathrm{M} \phi$ population in both healthy and diseased conditions.

Resident M $\phi$ s are indispensable for bone remodeling, as evidenced by the well-studied population of osteoclasts that drive bone resorption. Iron is implicated in the regulation of osteoclast function, such that iron release via Fpn is necessary for normal osteoclastogenesis and global skeletal homeostasis in mice (83). For instance, mice that lack Fpn activity in osteoclasts have accelerated osteoclastogenesis and skeletal resorption. A population of non-osteoclast resident bone $\mathrm{M} \phi \mathrm{s}$ (OsteoMacs) was characterized and shown to play a role in bone healing (84). Given this role in bone healing, it will be of great interest to determine whether OsteoMacs are iron-handling regulatory cells.

Pancreas. Both resident and recruited $\mathrm{M} \phi$ s have been implicated in pancreatic function and $\beta$ cell proliferation (85-88); however, we limit our discussion to the former in this Review. Populations of tissue-resident $\mathrm{M} \phi \mathrm{s}$ have been identified in both the endocrine (islets) and exocrine (interacinar stroma) pancreas (88). Exocrine $\mathrm{M} \phi$ s are proresolving in nature and appear resistant to inflammatory activation from diet-induced obesity (88). In contrast, islet $\mathrm{M} \phi \mathrm{s}$ - recently subcategorized into two populations, "intra-islet" and "peri-islet" (89) - may be inherently inflammatory in models of type 1 diabetes, where islet M $\phi$ s were shown to reside close to vascular beds, where they sample the islet milieu $(90,91)$. Furthermore, these islet $\mathrm{M} \phi$ s reportedly take up $\beta$ cell granules and present insulin peptides to autoimmune CD4+ $\mathrm{T}$ cells $(90,91)$. This may precede diabetic $\beta$ cell pathology, as the depletion of this population of $M \phi s$ in the NOD model improves diabetes outcomes (92). It is important to note that islet $\mathrm{M} \phi$ s have been studied mainly in the context of type 1 diabetes, and therefore, their role in other metabolic or pathological perturbations is less clear.

Lipotoxicity potentiates $\beta$ cell loss and is one mechanism that contributes to the pathogenesis of type 2 diabetes. Interestingly, in vitro, iron-depleted $\beta$ cells are more susceptible to palmitate-induced death than iron-sufficient cells (93). Palmitate decreases TfR1 expression in INS-1 $\beta$ cells, and gain- and loss-offunction studies reveal that overexpression of TfR1 in INS-1 $\beta$ cells protects against lipotoxicity-induced cell death, whereas TfR1 knockdown augments $\beta$ cell destruction (93). Thus, it is reasonable to posit that, in vivo, $M \phi s$ supply the required iron for $\beta$ cell defense in this context. An intriguing observation is that in genetic models of iron overload, excess iron appears to be preferentially stored in the exocrine, rather than endocrine, pancreas (94-97); however, this does not completely phenocopy human islets, which become iron loaded when challenged (98). Species-specific differences in the NTBI importer ZIP14 may in part explain these disparate findings (99). Nonetheless, additional studies are needed to understand the mechanisms behind intraregional differences in pancreatic iron handling and how the resident $\mathrm{M} \phi$ populations contribute to these differences.

Central nervous system. Impaired iron homeostasis in the CNS is coupled with neuroinflammation, oxidative stress, neurodegenerative disease pathology, and cognitive decline (100-103). Accordingly, iron must be tightly regulated on a cell-to-cell basis to ensure normal homeostatic function. Microglia, the resident $\mathrm{M} \phi s$ of the CNS, play an integral role in regulating brain iron levels (102, 104-109). Microglia express iron transport and storage proteins, including DMT1, TfR, ferritin, Fpn, and hepcidin, and acquire both holoTf and NTBI $(104,107)$. In particular, microglia can modulate cellular iron transport in response to their polarization state and extracellular milieu, exhibiting an enhanced preference for NTBI uptake and storage 
in proinflammatory M1-like conditions $(104,107)$. For example, exogenous exposure to TNF- $\alpha$ and TGF- $\beta$ increases microglial DMT1 expression and downregulates Fpn, leading to an accumulation of intracellular iron during an inflammatory response (110). On the other hand, antiinflammatory signaling is correlated with an increase in microglial import of Tf-bound iron that may be recycled during the reparative phase of an inflammatory response. Moreover, it has been suggested that shifting from a proinflammatory to an antiinflammatory state releases ferritin from M2-like microglial cells to assist in neuronal remyelination and repair after injury $(104,111)$.

While microglia adapt to regulate their own iron transport, these cells also play a central role in mediating iron levels among other cells in the brain. Healy et al. showed that increased ferritin expression and secretion occur preferentially in microglial cells in response to increased brain iron levels (112), and it is proposed that microglia accumulate and store iron more efficiently than other cells in the brain (113). Indeed, greater microglial iron tolerance may protect highly susceptible neurons from iron-induced toxicity and associated degeneration $(113,114)$. In addition to neurons, oligodendrocytes and astrocytes also respond to changes in microglial iron-handling phenotype, further highlighting the importance of these cells in regulating overall brain iron homeostasis $(115,116)$. For example, repressing microglial ferritin and hepcidin promotes oligodendrocyte-mediated healing after an ischemic insult (117), and microglial release of iron packaged in FtH under conditions of low parenchymal availability assists in functional oligodendrocyte cell survival $(106,107)$.

Of note, however, microglia are not impervious to iron overload-induced damage. Excessive iron can trigger microglial release of proinflammatory cytokines (such as TNF- $\alpha$, IL-6, and IL-1 $\beta$ ) and/or accentuate production of ROS that disturbs the function of adjacent cell types (101, 104, 114, 116). Microglia are iron loaded in active lesions of multiple sclerosis (118), and activated microglia are associated with increased iron uptake and retention in models of neurodegenerative disorders, such as Alzheimer's and Parkinson's disease $(107,109,114,118-120)$. It is clear that microglia play a critical role in the maintenance of brain iron homeostasis; however, more work is needed to elucidate the mechanisms and conditions in which microglial iron cycling contributes to and/or is protective against neural disorder.

Adipose tissue. Regulated control of local iron homeostasis is important in AT for two major reasons: (i) iron availability is necessary for normal adipogenesis (121); and (ii) excess fatty acids in adipocytes, particularly in the context of obesity, react with free iron and induce lipid peroxidation chain reactions, leading to aberrant oxidative stress (122). Along these lines, previous evidence shows that iron overload in adipocytes attenuates systemic insulin sensitivity via what appears to be an adiponectin-dependent mechanism (121, 123). Similarly, Gabrielsen et al. reported that adipocyte-targeted deletion of Fpn using the aP2-Cre mouse model triggers insulin resistance (121). In contrast, another study showed that adipocyte-specific Fpn deletion using the Adipoq-Cre recombinase mouse line does not cause a whole-body insulin-resistant phenotype, and feeding Adipoq-Cre Fpn-knockout mice a Western-type diet (supplemented with high-fructose corn syrup in drinking water, $42 \mathrm{~g} / \mathrm{L}$ ) did not increase adipocyte iron content compared with animals on a control diet (124). Notably, however, the aP2 promoter is also expressed in M $\phi$ s (125) and neurons (126), suggesting that the discrepant phenotype observed by Gabrielsen et al. (121) may be related to impaired neural and/or M $\phi$ iron cycling, although this postulate has not been formally tested.

Our group previously detected a population of iron-rich M $\phi$ s in AT — termed "MFe ${ }^{\text {hi" }}$ — in lean mice (18), with the remaining ATM $\phi$ s called " $M F e^{l o}$." These cells were isolated from the stromal vascular fraction of AT using magnetic columns, yielding ferromagnetic and non-ferromagnetic populations, respectively. Flow cytometric analysis of F4/80 and CD11b confirmed that the vast majority of ferromagnetic-positive cells were indeed M $\phi$ s (18). ATM $\phi s$ seemingly compensate for iron overload during a high-iron diet or intraperitoneal iron administration $(17,127)$, increasing their iron content while adipocyte iron concentrations remain stable in the face of iron overload (17). Importantly, $M F e^{\text {hi }}$ ATM $\phi$ s accumulate excess iron in AT while maintaining an M2-like polarization that is disturbed with diet-induced obesity (17). Interestingly, SpiC — the RPM $\phi$-associated transcription factor - is elevated in $\mathrm{MFe}^{\text {hi }}$ but not $\mathrm{MFe}^{\mathrm{lo}} \mathrm{ATM} \phi$ s from mice fed high-iron diets (17). It is tempting to speculate that $\mathrm{MFe}^{\mathrm{hi}} \mathrm{ATM} \phi$ s may have an origin distinct from that of their $\mathrm{MFe}^{\text {lo }}$ counterparts, yet this notion necessitates further testing. Various omics technologies (e.g., metabolomics, lipidomics), single-cell RNA sequencing, and/or lineage tracing studies using in vivo barcode generation (128) to characterize $\mathrm{MFe}^{\text {hi }}$ verses $\mathrm{MFe}^{\mathrm{lo}} \mathrm{ATM} \phi s$ would greatly advance our understanding of these distinct $\mathrm{M} \phi$ populations.

Skeletal and cardiac muscle. Iron is particularly important for cells with high mitochondrial activity, such as cardiomyocytes and skeletal muscle myocytes, which require iron not only for electron transport but for myoglobin production. Indeed, iron deficiency leads to myopathies in both cardiac and skeletal mus- 
cle (129). On the other hand, prior data reveal that iron overload in myocytes causes aberrant oxidative stress that contributes to muscle atrophy (130). Interestingly, $\mathrm{M} \phi s$ isolated from injured muscle express higher levels of haptoglobin, CD163, ferritin, and HO-1, suggesting that they contain the machinery to sequester myoglobin-iron released from damaged myocytes in response to acute injury (65). Five days following an initial insult, $\mathrm{M} \phi \mathrm{s}$ isolated from skeletal muscle upregulate Fpn, releasing iron and contributing to myofiber regeneration. The regenerative effect of these skeletal muscle $\mathrm{M} \phi \mathrm{s}$ is abrogated by targeted $F p n$ deletion (65), suggesting that iron cycling via $M \phi s$ in the context of muscle injury is necessary for appropriate activation of myogenic precursors and subsequent muscle healing. These data support the notion that local iron-handling $\mathrm{M} \phi \mathrm{s}$ buffer parenchymal cells from the toxic effects of superfluous iron, later supplying necessary iron in response to the tissue's needs in a highly coordinated manner. As a corollary, this temporal relationship between $\mathrm{M} \phi$ iron sequestration and iron donation in skeletal muscle injury mimics the M1-to-M2 transition seen during the tissue injury-to-repair paradigm.

Skin and wound healing. A previously underappreciated role for local iron cycling in skin homeostasis and repair has been identified. Using myeloid-targeted Fpn deletion, Recalcati et al. (64) reported that preventing $\mathrm{M} \phi$ iron cycling attenuates proliferation of skin epithelial cells and consequently impairs hair follicle growth, leading to transient alopecia in mice - an observation consistent with our mouse model of LysMCre-driven Fpn ablation (unpublished observations). These observations are notable given that the impaired hair growth and tissue repair were not attributable to systemic iron deficiency or anemia, supporting a direct local role for $\mathrm{M} \phi$ iron handling in regulating proper skin function (64). Another study revealed that impaired phenotypic switching from an M1 to an M2 polarization state led to iron overload in M1-like $\mathrm{M} \phi s$ that corresponded with impaired healing of chronic venous leg ulcers in mice and humans (131). Thus, one might surmise that M1-like M $\phi$ s have diminished iron turnover and decreased iron efflux, while $\mathrm{M} 2-$ like $\mathrm{M} \phi$ s retain the ability to recycle iron and partake in wound healing.

\section{Proposed model for iron-cycling $M \phi s$ in the regulation of tissue homeostasis}

In the previous section, we presented multiple examples of homeostatic regulation by iron-handling $M \phi s$. Based on the aforementioned examples, we suggest a simplified three-phase model (Figure 2) by which iron-cycling $\mathrm{M} \phi$ s maintain tissue homeostasis.

I. Injury. It is well appreciated that iron is important for immune cell proliferation and maturation, but iron is also essential for pathogens that compete for its capture. Indeed, in response to an injury or immune insult, parenchymal cells propagate stress signals, including PAMPs and damage-associated molecular patterns (DAMPs), that instruct M $\phi$ s to sequester iron during the injurious event. Iron is rapidly taken up by iron-handling $\mathrm{M} \phi \mathrm{s}$ to diminish iron-induced oxidative stress in parenchymal cells. Given that $\mathrm{M} \phi \mathrm{s}$ adopt an M1-like inflammatory phenotype during early stages of tissue injury, it is plausible that iNOS-catalyzed NO production by M $\phi s$ evokes parenchymal iron release via a DNIC-MRP1 efflux mechanism — akin to that used by TAMs (47-49) - that would then be rapidly taken up by iron-sequestering M $\phi$ s. Along with this increase in iron influx, $\mathrm{M} \phi \mathrm{s}$ exhibit decreased Fpn transcription and protein expression in response to PAMPs and DAMPs, reducing iron efflux $(23,39,132,133)$. M $\phi$ s may also produce hepcidin locally, particularly in response to bacterial signals, to reduce Fpn activity and iron efflux $(40,134,135)$. Recent evidence shows that resident $\mathrm{M} \phi \mathrm{s}$ cloak cells following mild (microlesion) but not substantial (macrolesion or multiple microlesions) injury, preventing rapid neutrophil-mediated destruction of the injured or dying cells (136). This physical $\mathrm{M} \phi$ barrier limits neutrophil contact with cellular debris and tempers neutrophil-induced inflammatory activation. It is tempting to speculate that iron cycling plays a role in this process, such that the cloaking $M \phi s$ take up excess iron from the injured parenchymal cell or from the extracellular space, sparing the cell from aberrant levels of oxidative stress.

II. Repair. Following acute stress, cell restoration includes many critical processes such as DNA synthesis, intracellular remodeling, and cellular proliferation. Accordingly, iron-handling M $\phi$ s supply the necessary iron required for these regenerative processes. The precise signaling mechanisms that trigger $\mathrm{M} \phi$ iron donation to parenchymal cells during the repair phase are not known and require further investigation. However, it is plausible that dampened proinflammatory signaling from injured parenchymal cells during initiation of the repair phase relieves $\mathrm{M} \phi$ inhibition of $F p n$ transcription, allowing reparative iron release from the $\mathrm{M} \phi$. Furthermore, antiinflammatory cytokines and pro-resolvins produced by $\mathrm{M} \phi \mathrm{s}$ may contribute to the transition from iron retention to iron donation, consistent with an M1- to M2-like M $\phi$ shift. 


\section{M $\phi$ Iron Handling During Tissue Injury}



PAMPs

DAMPs

Growth factors

Pro-resolvins

Inflammatory cytokines

Anti-inflammatory cytokines

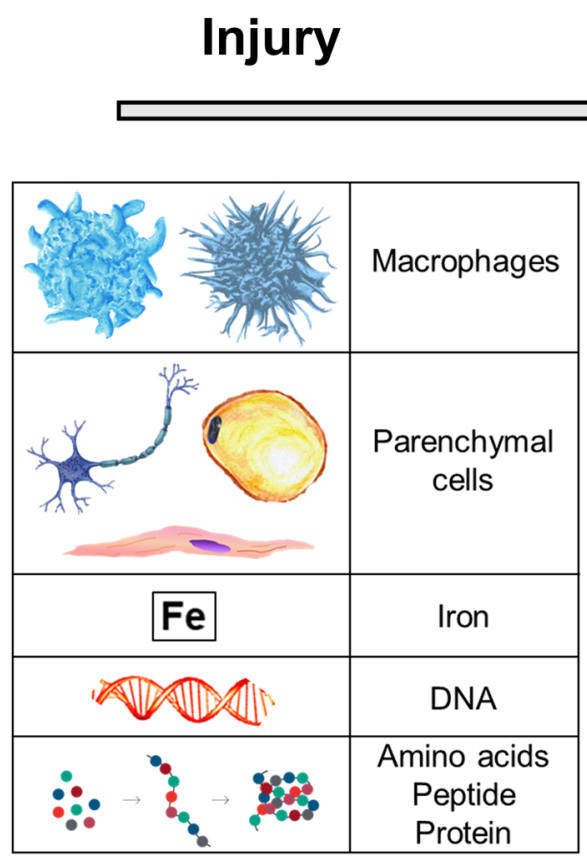

Repair

Resolution

\section{Time}

Figure 2. Proposed model by which resident iron-handling M $\phi s$ regulate tissue homeostasis during an insult. (1) Parenchymal cell injury initiates intracellular transduction signals that propagate transcriptional and nontranscriptional stress signals. (2) Local tissue-resident $M \phi s$ are recruited to the injured site. Since injured cells are more susceptible to iron-induced oxidative damage, $M \phi s$ sequester extracellular iron to decrease iron uptake in the injured parenchymal cell. LIP has been reported to increase in response to cell injury, which may promote iron efflux and $M \phi$ uptake. (3) A latency period follows, in which the $M \phi$ retains sequestered iron, allowing for parenchymal cell restoration. This phase of $M \phi$ iron retention may be aided by release of inflammatory cytokines and/or IFN responses that favor an iron-loaded $M \phi$ (i.e., suppression of Fpn-mediated iron export). Parenchymal cell repair requires iron for processes such as DNA synthesis. This utilization of parenchymal intracellular iron depletes ferritin stores. (4) The $M \Phi$ mobilizes and relinquishes iron for parenchymal cell repair. (5) An undefined termination signal communicates cell resolution and the $M \phi$ regresses from the site, completing the homeostatic circuit and maintaining local iron balance. 
III. Resolution. The transition between parenchymal cell repair and cell resolution is a multifaceted process whereby $\mathrm{M} \phi$ s play a crucial role in clearing debris and secreting pro-resolvin mediators and antiinflammatory cytokines (137). In addition, we believe that tightly regulated iron donation by $M \phi s$ is a critical piece of this repair-to-resolution process. Indeed, loss of $\mathrm{M} \phi$ iron donation delays or impairs wound healing in mice (64, $65,83)$. Thus, we propose that $M \phi s$ continually supply iron to cells undergoing repair until cell regeneration/ repair is completed or near completion. Thereafter, a balance in iron trafficking between $\mathrm{M} \phi \mathrm{s}$ and parenchymal cells is achieved, thereby completing the circuit and restoring a state of tissue homeostasis.

To summarize this basic homeostatic circuit, resident $M \phi s$ first protect cells from excessive iron-induced oxidative damage and later supply iron for restorative processes in a highly coordinated and time-dependent manner. This model is consistent with the notion proposed by Cairo et al., who suggested that, "because iron retention by inflammatory $\mathrm{M} \phi s$ contributes to pathogen control during the acute phase of inflammation, the ability of alternatively-activated $M \phi s$ to donate iron could provide a relevant contribution to tissue repair in the resolution phase" (138). These are important observations, particularly in regard to the transition states between injurious events (139). That is, during the inflammatory phase of an acute injury, $\mathrm{M} \phi$ iron sequestration may advantageously protect the injured cell from iron-induced oxidative damage, whereas during resolution, the $\mathrm{M} \phi s$ must relinquish labile iron required by the previously injured parenchymal cell for repair. It is plausible that the capacity of $M \phi s$ to transition smoothly between these states (i.e., iron retention versus iron donation) dictates whole-tissue homeostasis.

Considerations. Several considerations should be noted in regard to the abovementioned model. First, the model assumes a normal homeostatic starting point (i.e., healthy $\mathrm{M} \phi$ and parenchymal cell phenotype). For instance, iron-overloaded M $\phi$ s have dampened antimicrobial effector function (140), possibly due to the inability to sequester parenchymal iron in an already iron-loaded state. Thus, various pathologies will likely disturb the circuit at one or more steps. Indeed, patients with type 1 hemochromatosis exhibit iron-deficient M $\phi$ s despite whole-body iron overload, and they are more susceptible to infections (141). Furthermore, in atherosclerosis, $\mathrm{Hb}$-handling $\mathrm{M} \phi s$ have been reported to take on both pro- and antiatherogenic roles (reviewed in ref. 142). Thus, there seems to be a complex temporal relationship between iron trafficking and disease pathogenicity. Second, in addition to pathological conditions, natural aging likely influences $M \phi$ iron handling. Indeed, in vivo and in vitro evidence reveals that aging disrupts $\mathrm{M} \phi$ phagocytic activity, polarization, and wound healing capacity (143), all of which are influenced by iron status. Third, our current understanding of $\mathrm{M} \phi$ iron handling is largely limited to in vitro studies that employ extreme iron loads and chelation approaches that may not directly mimic the more nuanced in vivo environment. Hence, to further reveal physiologically meaningful conclusions about how $\mathrm{M} \phi$ iron handling regulates tissue function, novel in vivo strategies that specifically target $\mathrm{M} \phi$ iron handling are needed. Notwithstanding these considerations, a better understanding of the mechanisms governing the transition states between injury, repair, and resolution could allow discovery of drug targets or development of alternative therapeutic interventions.

Outstanding questions. Despite the advances in our understanding of the functions and phenotypes of iron-handling $\mathrm{M} \phi s$, multiple questions remain unanswered. Do all iron-cycling $M \phi s$ arise from a similar lineage? For example, splenic RPM $\phi$ s are yolk sac derived and require the SpiC transcription factor for their development (55). Is this inherent to all tissue-resident iron-handling Mфs? What are the molecular mechanisms that govern iron cycling? That is, what signals $M \phi s$ to sequester iron rather than to release iron in the homeostatic circuit? On a macro scale, it is clear that the hepcidin/Fpn axis is an important regulator of iron cycling; yet, this may not explain tissue-specific $\mathrm{M} \phi$-mediated iron cycling activity, where local activity may operate independently from systemic hormonal cues. Indeed, alveolar M $\phi$ s were recently implicated in lung iron trafficking and may exhibit some independence from the hepcidin/Fpn axis (144-146), though the cellular mechanisms require additional study. Is it strictly the $\mathrm{M} \phi$ polarization and/or activation state that predicts iron-cycling activity; or does iron cycling itself dictate the activation and/or polarization state? Are there distinct cell-surface markers or intracellular genes inherent to iron-cycling $\mathrm{M} \phi s$ that could be therapeutic targets? Single-cell RNA sequencing and omics technologies in iron-handling M $\phi s$ would greatly advance our understanding of this unique population. Finally, what mechanism(s) govern whether iron-handling $\mathrm{M} \phi$ s contribute to, or protect against, disease status?

\section{Concluding remarks}

Iron affects every cell in a fundamental fashion, linking both metabolism and host defense. Hence, mechanisms and intercellular circuits must be in place to tightly regulate and fine-tune iron flux. Accumulating 
data indicate that in addition to patrolling for changes in systemic iron levels, resident $\mathrm{M} \phi \mathrm{s}$ are critical in handling iron at the local tissue level. In this Review, we have compiled evidence from various tissues and organs to suggest that tissue-resident $\mathrm{M} \phi \mathrm{s}$ are ferrostats that play a critical role in maintaining tissue microenvironments to regulate both local and systemic homeostasis. It is likely that many other organs and tissues not discussed here are modulated by resident iron-cycling M $\phi s$. Thus, our Review represents just the tip of the iceberg of potential knowledge about how $M \phi s$ contribute to tissue iron regulation. Notwithstanding the tissue of interest, we propose that the capacity of iron-handling $M \phi s$ to transition smoothly between iron-retaining and iron-donating states largely dictates tissue homeostasis. In conclusion, numerous exciting revelations pertaining to tissue iron homeostasis and $\mathrm{M} \phi$ function have arisen in recent years; however, additional work is required to uncover the underlying intrinsic and extrinsic mechanisms by which the macrophage ferrostat coordinates cellular, tissue, and organ function.

\section{Acknowledgments}

AHH is supported by NIH grant R01-DK121520. NCW is supported by an American Physiological Society Postdoctoral Fellowship. KMV is supported by an NIH Molecular Endocrinology Training Grant (DK007563). We gratefully acknowledge Anna Bright for artwork featured in the figures; and Kendra Oliver for technical assistance performed as part of the ArtLab \& Vanderbilt Institute for Infection, Immunology, and Inflammation (VI4) Artist-in-Residence pilot program at Vanderbilt University and Vanderbilt University Medical Center.

Address correspondence to: Alyssa H. Hasty, 702 Light Hall, Nashville, Tennessee, 37232, USA. Phone: 615.322.5177; Email: alyssa.hasty@vanderbilt.edu.

1. Musallam KM, Taher AT. Iron deficiency beyond erythropoiesis: should we be concerned? Curr Med Res Opin. 2018;34(1):81-93

2. von Haehling S, Jankowska EA, van Veldhuisen DJ, Ponikowski P, Anker SD. Iron deficiency and cardiovascular disease. Nat Rev Cardiol. 2015;12(11):659-669.

3. Fernandez-Real JM, Manco M. Effects of iron overload on chronic metabolic diseases. Lancet Diabetes Endocrinol. 2014;2(6):513-526.

4. Ward RJ, Zucca FA, Duyn JH, Crichton RR, Zecca L. The role of iron in brain ageing and neurodegenerative disorders. Lancet Neurol. 2014;13(10):1045-1060.

5. Pantopoulos K, Porwal SK, Tartakoff A, Devireddy L. Mechanisms of mammalian iron homeostasis. Biochemistry. 2012;51(29):5705-5724.

6. Muckenthaler MU, Rivella S, Hentze MW, Galy B. A red carpet for iron metabolism. Cell. 2017;168(3):344-361.

7. Sukhbaatar N, Weichhart T. Iron regulation: macrophages in control. Pharmaceuticals (Basel). 2018;11(4):E137.

8. Nairz M, Theurl I, Swirski FK, Weiss G. "Pumping iron" - how macrophages handle iron at the systemic, microenvironmental, and cellular levels. Pflugers Arch. 2017;469(3-4):397-418.

9. Klei TR, Meinderts SM, van den Berg TK, van Bruggen R. From the cradle to the grave: the role of macrophages in erythropoiesis and erythrophagocytosis. Front Immunol. 2017;8:73.

10. Korolnek T, Hamza I. Macrophages and iron trafficking at the birth and death of red cells. Blood. 2015;125(19):2893-2897.

11. Murray PJ. Macrophage polarization. Annu Rev Physiol. 2017;79:541-566.

12. Zhou D, et al. Macrophage polarization and function with emphasis on the evolving roles of coordinated regulation of cellular signaling pathways. Cell Signal. 2014;26(2):192-197.

13. Ginhoux F, Guilliams M. Tissue-resident macrophage ontogeny and homeostasis. Immunity. 2016;44(3):439-449.

14. Davies LC, Taylor PR. Tissue-resident macrophages: then and now. Immunology. 2015;144(4):541-548.

15. Caslin HL, Hasty AH. Extrinsic and intrinsic immunometabolism converge: perspectives on future research and therapeutic development for obesity. Curr Obes Rep. 2019;8(3):210-219.

16. Fantin A, et al. Tissue macrophages act as cellular chaperones for vascular anastomosis downstream of VEGF-mediated endothelial tip cell induction. Blood. 2010;116(5):829-840.

17. Hubler MJ, Erikson KM, Kennedy AJ, Hasty AH. MFe ${ }^{\text {hi }}$ adipose tissue macrophages compensate for tissue iron perturbations in mice. Am J Physiol, Cell Physiol. 2018;315(3):C319-C329.

18. Orr JS, et al. Obesity alters adipose tissue macrophage iron content and tissue iron distribution. Diabetes. 2014;63(2):421-432.

19. Cherayil BJ. The role of iron in the immune response to bacterial infection. Immunol Res. 2011;50(1):1-9.

20. Ganz T, Nemeth E. Iron homeostasis in host defence and inflammation. Nat Rev Immunol. 2015;15(8):500-510.

21. Wang CY, Babitt JL. Liver iron sensing and body iron homeostasis. Blood. 2019;133(1):18-29.

22. Knutson MD. Iron transport proteins: gateways of cellular and systemic iron homeostasis. J Biol Chem. 2017;292(31):12735-12743.

23. Drakesmith H, Nemeth E, Ganz T. Ironing out ferroportin. Cell Metab. 2015;22(5):777-787.

24. Philpott CC, Jadhav S. The ins and outs of iron: escorting iron through the mammalian cytosol. Free Radic Biol Med. 2019;133:112-117.

25. Shvartsman M, Ioav Cabantchik Z. Intracellular iron trafficking: role of cytosolic ligands. Biometals. 2012;25(4):711-723.

26. Arosio P, Ingrassia R, Cavadini P. Ferritins: a family of molecules for iron storage, antioxidation and more. Biochim Biophys Acta. 2009;1790(7):589-599. 
27. Cozzi A, Corsi B, Levi S, Santambrogio P, Albertini A, Arosio P. Overexpression of wild type and mutated human ferritin H-chain in HeLa cells: in vivo role of ferritin ferroxidase activity. J Biol Chem. 2000;275(33):25122-25129.

28. Ferreira C, et al. H ferritin knockout mice: a model of hyperferritinemia in the absence of iron overload. Blood. 2001;98(3):525-532.

29. Cozzi A, et al. Human L-ferritin deficiency is characterized by idiopathic generalized seizures and atypical restless leg syndrome. J Exp Med. 2013;210(9):1779-1791.

30. Levi S, Rovida E. Neuroferritinopathy: from ferritin structure modification to pathogenetic mechanism. Neurobiol Dis. 2015;81:134-143

31. Tang M, Chen Z, Wu D, Chen L. Ferritinophagy/ferroptosis: iron-related newcomers in human diseases. J Cell Physiol. 2018;233(12):9179-9190.

32. Bellelli R, et al. NCOA4 Deficiency impairs systemic iron homeostasis. Cell Rep. 2016;14(3):411-421.

33. Lahiri V, Hawkins WD, Klionsky DJ. Watch what you (self-) eat: autophagic mechanisms that modulate metabolism. Cell Metab. 2019;29(4):803-826.

34. Quiles Del Rey M, Mancias JD. NCOA4-mediated ferritinophagy: a potential link to neurodegeneration. Front Neurosci. 2019;13:238.

35. Santana-Codina N, Mancias JD. The role of NCOA4-mediated ferritinophagy in health and disease. Pharmaceuticals (Basel). 2018;11(4):E114.

36. Musci G, Polticelli F, Bonaccorsi di Patti MC. Ceruloplasmin-ferroportin system of iron traffic in vertebrates. World J Biol Chem 2014;5(2):204-215.

37. Zhang Z, et al. Ferroportin1 deficiency in mouse macrophages impairs iron homeostasis and inflammatory responses. Blood. 2011;118(7):1912-1922.

38. Donovan A, et al. The iron exporter ferroportin/Slc40a1 is essential for iron homeostasis. Cell Metab. 2005;1(3):191-200.

39. Abreu R, Quinn F, Giri PK. Role of the hepcidin-ferroportin axis in pathogen-mediated intracellular iron sequestration in human phagocytic cells. Blood Adv. 2018;2(10):1089-1100.

40. Chung B, Chaston T, Marks J, Srai SK, Sharp PA. Hepcidin decreases iron transporter expression in vivo in mouse duodenum and spleen and in vitro in THP-1 macrophages and intestinal Caco-2 cells. J Nutr. 2009;139(8):1457-1462.

41. Chaston T, et al. Evidence for differential effects of hepcidin in macrophages and intestinal epithelial cells. Gut. 2008;57(3):374-382.

42. Truman-Rosentsvit M, et al. Ferritin is secreted via 2 distinct nonclassical vesicular pathways. Blood. 2018;131(3):342-352.

43. Meyron-Holtz EG, Moshe-Belizowski S, Cohen LA. A possible role for secreted ferritin in tissue iron distribution. J Neural Transm (Vienna). 2011;118(3):337-347.

44. Cohen LA, et al. Serum ferritin is derived primarily from macrophages through a nonclassical secretory pathway. Blood. 2010;116(9):1574-1584.

45. Leimberg JM, Konijn AM, Fibach E. Macrophages promote development of human erythroid precursors in transferrin-free culture medium. Hematology. 2005;10(1):73-76

46. Sheokand N, et al. Reverse overshot water-wheel retroendocytosis of apotransferrin extrudes cellular iron. J Cell Sci. 2016;129(4):843-853.

47. Lok HC, Sahni S, Jansson PJ, Kovacevic Z, Hawkins CL, Richardson DR. A nitric oxide storage and transport system that protects activated macrophages from endogenous nitric oxide cytotoxicity. J Biol Chem. 2016;291(53):27042-27061.

48. Lok HC, et al. Nitric oxide storage and transport in cells are mediated by glutathione S-transferase P1-1 and multidrug resistance protein 1 via dinitrosyl iron complexes. J Biol Chem. 2012;287(1):607-618.

49. Watts RN, Hawkins C, Ponka P, Richardson DR. Nitrogen monoxide (NO)-mediated iron release from cells is linked to NO-induced glutathione efflux via multidrug resistance-associated protein 1. Proc Natl Acad Sci U S A. 2006;103(20):7670-7675.

50. Coffey R, Ganz T. Iron homeostasis: an anthropocentric perspective. J Biol Chem. 2017;292(31):12727-12734.

51. de Back DZ, Kostova EB, van Kraaij M, van den Berg TK, van Bruggen R. Of macrophages and red blood cells; a complex love story. Front Physiol. 2014;(5):9.

52. Donegan RK, Moore CM, Hanna DA, Reddi AR. Handling heme: the mechanisms underlying the movement of heme within and between cells. Free Radic Biol Med. 2019;133:88-100.

53. White C, et al. HRG1 is essential for heme transport from the phagolysosome of macrophages during erythrophagocytosis. Cell Metab. 2013;17(2):261-270.

54. Hower V, et al. A general map of iron metabolism and tissue-specific subnetworks. Mol Biosyst. 2009;5(5):422-443

55. Kohyama M, et al. Role for Spi-C in the development of red pulp macrophages and splenic iron homeostasis. Nature. 2009;457(7227):318-321.

56. Kovtunovych G, et al. Wild-type macrophages reverse disease in heme oxygenase 1-deficient mice. Blood. 2014;124(9):1522-1530.

57. Chow A, et al. CD169+ macrophages provide a niche promoting erythropoiesis under homeostasis and stress. Nat Med. 2013;19(4):429-436

58. Corna G, et al. Polarization dictates iron handling by inflammatory and alternatively activated macrophages. Haematologica. 2010;95(11):1814-1822.

59. Recalcati S, et al. Differential regulation of iron homeostasis during human macrophage polarized activation. Eur J Immunol. 2010;40(3):824-835.

60. Agoro R, Mura C. Inflammation-induced up-regulation of hepcidin and down-regulation of ferroportin transcription are dependent on macrophage polarization. Blood Cells Mol Dis. 2016;61:16-25.

61. Gaetano C, Massimo L, Alberto M. Control of iron homeostasis as a key component of macrophage polarization. Haematologica. 2010;95(11):1801-1803.

62. Davies LC, Jenkins SJ, Allen JE, Taylor PR. Tissue-resident macrophages. Nat Immunol. 2013;14(10):986-995.

63. Hashimoto D, et al. Tissue-resident macrophages self-maintain locally throughout adult life with minimal contribution from circulating monocytes. Immunity. 2013;38(4):792-804.

64. Recalcati S, et al. Macrophage ferroportin is essential for stromal cell proliferation in wound healing. Haematologica. 2019;104(1):47-58.

65. Corna $\mathrm{G}$, et al. The repair of skeletal muscle requires iron recycling through macrophage ferroportin. J Immunol. 
2016;197(5):1914-1925

66. Kroner A, Greenhalgh AD, Zarruk JG, Passos Dos Santos R, Gaestel M, David S. TNF and increased intracellular iron alter macrophage polarization to a detrimental M1 phenotype in the injured spinal cord. Neuron. 2014;83(5):1098-1116.

67. Bories $\mathrm{G}$, et al. Liver $\mathrm{X}$ receptor activation stimulates iron export in human alternative macrophages. Circ Res. 2013;113(11):1196-1205.

68. Bouwens L, Baekeland M, De Zanger R, Wisse E. Quantitation, tissue distribution and proliferation kinetics of Kupffer cells in normal rat liver. Hepatology. 1986;6(4):718-722.

69. Hoeffel G, et al. C-Myb(+) erythro-myeloid progenitor-derived fetal monocytes give rise to adult tissue-resident macrophages. Immunity. 2015;42(4):665-678.

70. Gomez Perdiguero E, et al. Tissue-resident macrophages originate from yolk-sac-derived erythro-myeloid progenitors. Nature. 2015;518(7540):547-551.

71. Sheng J, Ruedl C, Karjalainen K. Most tissue-resident macrophages except microglia are derived from fetal hematopoietic stem cells. Immunity. 2015;43(2):382-393.

72. Li P, He K, Li J, Liu Z, Gong J. The role of Kupffer cells in hepatic diseases. Mol Immunol. 2017;85:222-229.

73. Scott CL, et al. Bone marrow-derived monocytes give rise to self-renewing and fully differentiated Kupffer cells. Nat Commun. 2016;7:10321.

74. Song M, et al. Kupffer cell depletion protects against the steatosis, but not the liver damage, induced by marginal-copper, high-fructose diet in male rats. Am J Physiol Gastrointest Liver Physiol. 2015;308(11):G934-G945.

75. Theurl I, et al. On-demand erythrocyte disposal and iron recycling requires transient macrophages in the liver. Nat Med. 2016;22(8):945-951.

76. Theurl M, et al. Kupffer cells modulate iron homeostasis in mice via regulation of hepcidin expression. $J$ Mol Med. 2008;86(7):825-835

77. Kovtunovych G, Eckhaus MA, Ghosh MC, Ollivierre-Wilson H, Rouault TA. Dysfunction of the heme recycling system in heme oxygenase 1-deficient mice: effects on macrophage viability and tissue iron distribution. Blood. 2010;116(26):6054-6062

78. Haldar M, et al. Heme-mediated SPI-C induction promotes monocyte differentiation into iron-recycling macrophages. Cell. 2014;156(6):1223-1234.

79. Kurotaki D, et al. CSF-1-dependent red pulp macrophages regulate CD4 T cell responses. J Immunol. 2011;186(4):2229-2237.

80. Jacobsen RN, Perkins AC, Levesque JP. Macrophages and regulation of erythropoiesis. Curr Opin Hematol. 2015;22(3):212-219.

81. Ramos $\mathrm{P}$, et al. Macrophages support pathological erythropoiesis in polycythemia vera and $\beta$-thalassemia. Nat Med. 2013;19(4):437-445

82. Li W, et al. Identification and transcriptome analysis of erythroblastic island macrophages. Blood. 2019;134(5):480-491.

83. Wang L, et al. Deletion of ferroportin in murine myeloid cells increases iron accumulation and stimulates osteoclastogenesis in vitro and in vivo. J Biol Chem. 2018;293(24):9248-9264.

84. Chang MK, et al. Osteal tissue macrophages are intercalated throughout human and mouse bone lining tissues and regulate osteoblast function in vitro and in vivo. J Immunol. 2008;181(2):1232-1244.

85. Riley KG, et al. Macrophages are essential for CTGF-mediated adult $\beta$-cell proliferation after injury. Mol Metab. 2015;4(8):584-591

86. Xiao X, et al. M2 macrophages promote beta-cell proliferation by up-regulation of SMAD7. Proc Natl Acad Sci U S A. 2014;111(13):E1211-E1220.

87. Brissova $\mathrm{M}$, et al. Islet microenvironment, modulated by vascular endothelial growth factor-A signaling, promotes $\beta$ cell regeneration. Cell Metab. 2014;19(3):498-511.

88. Calderon B, et al. The pancreas anatomy conditions the origin and properties of resident macrophages. $J$ Exp Med. 2015;212(10):1497-1512.

89. Ying W, et al. Expansion of islet-resident macrophages leads to inflammation affecting $\beta$ cell proliferation and function in obesity. Cell Metab. 2019;29(2):457-474.e5.

90. Zinselmeyer BH, Vomund AN, Saunders BT, Johnson MW, Carrero JA, Unanue ER. The resident macrophages in murine pancreatic islets are constantly probing their local environment, capturing beta cell granules and blood particles. Diabetologia. 2018;61(6):1374-1383

91. Calderon B, Carrero JA, Miller MJ, Unanue ER. Cellular and molecular events in the localization of diabetogenic T cells to islets of Langerhans. Proc Natl Acad Sci U S A. 2011;108(4):1561-1566.

92. Carrero JA, et al. Resident macrophages of pancreatic islets have a seminal role in the initiation of autoimmune diabetes of NOD mice. Proc Natl Acad Sci U S A. 2017;114(48):E10418-E10427.

93. Jung IR, et al. Involvement of iron depletion in palmitate-induced lipotoxicity of beta cells. Mol Cell Endocrinol. 2015;407:74-84

94. Chen M, et al. Ceruloplasmin and hephaestin jointly protect the exocrine pancreas against oxidative damage by facilitating iron efflux. Redox Biol. 2018;17:432-439.

95. Lunova $\mathrm{M}$, et al. Hepcidin knockout mice spontaneously develop chronic pancreatitis owing to cytoplasmic iron overload in acinar cells. $J$ Pathol. 2017;241(1):104-114.

96. Altamura S, et al. Resistance of ferroportin to hepcidin binding causes exocrine pancreatic failure and fatal iron overload. Cell Metab. 2014;20(2):359-367.

97. Kato T, Daimon M, Kawanami T, Ikezawa Y, Sasaki H, Maeda K. Islet changes in hereditary ceruloplasmin deficiency. Hum Pathol. 1997;28(4):499-502.

98. Coffey R, Knutson MD. The plasma membrane metal-ion transporter ZIP14 contributes to nontransferrin-bound iron uptake by human $\beta$-cells. Am J Physiol, Cell Physiol. 2017;312(2):C169-C175.

99. Nam H, et al. ZIP14 and DMT1 in the liver, pancreas, and heart are differentially regulated by iron deficiency and overload: implications for tissue iron uptake in iron-related disorders. Haematologica. 2013;98(7):1049-1057.

100. Ndayisaba A, Kaindlstorfer C, Wenning GK. Iron in neurodegeneration — cause or consequence? Front Neurosci. 2019;13:180

101. Yauger YJ, Bermudez S, Moritz KE, Glaser E, Stoica B, Byrnes KR. Iron accentuated reactive oxygen species release by NADPH oxidase in activated microglia contributes to oxidative stress in vitro. J Neuroinflammation. 2019;16(1):41

102. Sfera A, Gradini R, Cummings M, Diaz E, Price AI, Osorio C. Rusty microglia: trainers of innate immunity in Alzheimer's 
disease. Front Neurol. 2018;9:1062.

103. Kim GH, Kim JE, Rhie SJ, Yoon S. The role of oxidative stress in neurodegenerative diseases. Exp Neurobiol. 2015;24(4):325-340. 104. Nnah IC, Wessling-Resnick M. Brain iron homeostasis: a focus on microglial iron. Pharmaceuticals (Basel). 2018;11(4):E129. 105. Vela D. The dual role of hepcidin in brain iron load and inflammation. Front Neurosci. 2018;12:740.

106. Puy V, et al. Predominant role of microglia in brain iron retention in Sanfilippo syndrome, a pediatric neurodegenerative disease. Glia. 2018;66(8):1709-1723.

107. McCarthy RC, Sosa JC, Gardeck AM, Baez AS, Lee CH, Wessling-Resnick M. Inflammation-induced iron transport and metabolism by brain microglia. J Biol Chem. 2018;293(20):7853-7863.

108. Regen F, Hellmann-Regen J, Costantini E, Reale M. Neuroinflammation and Alzheimer's disease: implications for microglial activation. Curr Alzheimer Res. 2017;14(11):1140-1148.

109. Zeineh MM, Chen Y, Kitzler HH, Hammond R, Vogel H, Rutt BK. Activated iron-containing microglia in the human hippocampus identified by magnetic resonance imaging in Alzheimer disease. Neurobiol Aging. 2015;36(9):2483-2500.

110. Rathore KI, Redensek A, David S. Iron homeostasis in astrocytes and microglia is differentially regulated by TNF- $\alpha$ and TGFB1. Glia. 2012;60(5):738-750.

111. McCarthy RC, Sosa JC, Gardeck AM, Baez AS, Lee CH, Wessling-Resnick M. Inflammation-induced iron transport and metabolism by brain microglia. J Biol Chem. 2018;293(20):7853-7863.

112. Healy S, McMahon J, Owens P, FitzGerald U. Significant glial alterations in response to iron loading in a novel organotypic hippocampal slice culture model. Sci Rep. 2016;6:36410.

113. Bishop GM, Dang TN, Dringen R, Robinson SR. Accumulation of non-transferrin-bound iron by neurons, astrocytes, and microglia. Neurotox Res. 2011;19(3):443-451.

114. Xu H, Wang Y, Song N, Wang J, Jiang H, Xie J. New progress on the role of glia in iron metabolism and iron-induced degeneration of dopamine neurons in parkinson's disease. Front Mol Neurosci. 2017;10:455.

115. Wang HF, et al. Effect of glial cells on remyelination after spinal cord injury. Neural Regen Res. 2017;12(10):1724-1732.

116. Zhang W, et al. Role and mechanism of microglial activation in iron-induced selective and progressive dopaminergic neurodegeneration. Mol Neurobiol. 2014;49(3):1153-1165

117. Belaidi AA, Bush AI. Iron neurochemistry in Alzheimer's disease and Parkinson's disease: targets for therapeutics. J Neurochem. 2016;139 Suppl 1:179-197.

118. Gillen KM, Mubarak M, Nguyen TD, Pitt D. Significance and in vivo detection of iron-laden microglia in white matter multiple sclerosis lesions. Front Immunol. 2018;9:255.

119. McIntosh A, et al. Iron accumulation in microglia triggers a cascade of events that leads to altered metabolism and compromised function in APP/PS1 mice. Brain Pathol. 2019;29(5):606-621.

120. Holland R, et al. Inflammatory microglia are glycolytic and iron retentive and typify the microglia in APP/PS1 mice. Brain Behav Immun. 2018;68:183-196

121. Gabrielsen JS, et al. Adipocyte iron regulates adiponectin and insulin sensitivity. J Clin Invest. 2012;122(10):3529-3540.

122. Winterbourn CC. Toxicity of iron and hydrogen peroxide: the Fenton reaction. Toxicol Lett. 1995;82-83:969-974.

123. Zheng J, Chen M, Liu G, Xu E, Chen H. Ablation of hephaestin and ceruloplasmin results in iron accumulation in adipocytes and type 2 diabetes. FEBS Lett. 2018;592(3):394-401.

124. Britton L, et al. Ferroportin expression in adipocytes does not contribute to iron homeostasis or metabolic responses to a high calorie diet. Cell Mol Gastroenterol Hepatol. 2018;5(3):319-331.

125. Lee KY, et al. Lessons on conditional gene targeting in mouse adipose tissue. Diabetes. 2013;62(3):864-874.

126. Martens K, Bottelbergs A, Baes M. Ectopic recombination in the central and peripheral nervous system by aP2/FABP4-Cre mice: implications for metabolism research. FEBS Lett. 2010;584(5):1054-1058.

127. Gotardo ÉM, Caria CR, de Oliveira CC, Rocha T, Ribeiro ML, Gambero A. Effects of iron supplementation in mice with hypoferremia induced by obesity. Exp Biol Med (Maywood). 2016;241(18):2049-2055.

128. Kalhor R, et al. Developmental barcoding of whole mouse via homing CRISPR. Science. 2018;361(6405):eaat9804.

129. Xu W, Barrientos T, Andrews NC. Iron and copper in mitochondrial diseases. Cell Metab. 2013;17(3):319-328.

130. Ikeda Y, et al. Iron-induced skeletal muscle atrophy involves an Akt-forkhead box O3-E3 ubiquitin ligase-dependent pathway. $J$ Trace Elem Med Biol. 2016;35:66-76.

131. Sindrilaru A, et al. An unrestrained proinflammatory M1 macrophage population induced by iron impairs wound healing in humans and mice. J Clin Invest. 2011;121(3):985-997.

132. Guida C, et al. A novel inflammatory pathway mediating rapid hepcidin-independent hypoferremia. Blood. 2015;125(14):2265-2275.

133. Deschemin JC, Vaulont S. Role of hepcidin in the setting of hypoferremia during acute inflammation. PLoS One. 2013;8(4):e61050

134. Peyssonnaux C, Zinkernagel AS, Datta V, Lauth X, Johnson RS, Nizet V. TLR4-dependent hepcidin expression by myeloid cells in response to bacterial pathogens. Blood. 2006;107(9):3727-3732.

135. Theurl I, et al. Autocrine formation of hepcidin induces iron retention in human monocytes. Blood. 2008;111(4):2392-2399.

136. Uderhardt S, Martins AJ, Tsang JS, Lämmermann T, Germain RN. Resident macrophages cloak tissue microlesions to prevent neutrophil-driven inflammatory damage. Cell. 2019;177(3):541-555.e17.

137. Vannella KM, Wynn TA. Mechanisms of organ injury and repair by macrophages. Annu Rev Physiol. 2017;79:593-617.

138. Cairo G, Recalcati S, Mantovani A, Locati M. Iron trafficking and metabolism in macrophages: contribution to the polarized phenotype. Trends Immunol. 2011;32(6):241-247.

139. Patsalos A, et al. The BACH1-HMOX1 regulatory axis is indispensable for proper macrophage subtype specification and skeletal muscle regeneration. J Immunol. 2019;203(6):1532-1547.

140. Soares MP, Weiss G. The Iron age of host-microbe interactions. EMBO Rep. 2015;16(11):1482-1500.

141. Cairo G, Recalcati S, Montosi G, Castrusini E, Conte D, Pietrangelo A. Inappropriately high iron regulatory protein activity in monocytes of patients with genetic hemochromatosis. Blood. 1997;89(7):2546-2553.

142. Cornelissen A, Guo L, Sakamoto A, Virmani R, Finn AV. New insights into the role of iron in inflammation and atherosclerosis. EBioMedicine. 2019;47:598-606. 
143.Linehan E, Fitzgerald DC. Ageing and the immune system: focus on macrophages. Eur J Microbiol Immunol (Bp). 2015;5(1):14-24. 144. Deschemin JC, Mathieu JRR, Zumerle S, Peyssonnaux C, Vaulont S. Pulmonary iron homeostasis in hepcidin knockout mice. Front Physiol. 2017;8:804.

145. Neves J, et al. Disruption of the hepcidin/ferroportin regulatory system causes pulmonary iron overload and restrictive lung disease. EBioMedicine. 2017;20:230-239.

146. Nguyen NB, Callaghan KD, Ghio AJ, Haile DJ, Yang F. Hepcidin expression and iron transport in alveolar macrophages. Am J Physiol Lung Cell Mol Physiol. 2006;291(3):L417-L425. 